\title{
No Knowledge to Waste - Learning more about Processes
}

\author{
Ir. K. Loossens \& Ir. G. Van Houtte \\ Expert Systems Applications Development Group \\ Department of Chemical Engineering \\ Katholieke Universiteit Leuven \\ de Croylaan 46, B-3001 Heverlee, Belgium \\ Koen.Loossens@cit.kuleuven.ac.be \\ Guy.VanHoutte@cit.kuleuven.ac.be
}

\begin{abstract}
Automating your process is one thing, using your investment in an optimal way is another. Automation systems provide you with a lot of process data, which should lead to a better understanding of the process. This is seldom the case, the culprit being an overdose of information. As a result, a lot of useful information remains hidden in these data. In this article we present a system that is capable of distilling knowledge out of process data. Based on the current process situation and using information about previous operations, knowledge is generated on the fly. As a result existing knowledge can be validated and hidden process characteristics can be revealed. Without expensive expert system technology, the engineer can maximally use the information that is being logged in the plant and gradually expand his know-how of the process. He can then put this knowledge at the disposal of the operators who can use this to better manage the process. The proposed system is generally applicable throughout the process industry. Two industrial examples are referenced to: a waste paper plant (continuous operation), and a pulp factory (batch operation of digesters).
\end{abstract}

\section{INTRODUCTION}

Today's automation systems are capable of measuring and collecting every desired detail about the production process. This massive amount of information should lead to a better understanding and management of the process. However, the problem is how to cash in on this information as to get the most out of your investments in an automation system. An engineer can attempt to uncover some knowledge with statistical tools and modelling approaches but often they are confronted with a process that is too complicated and too less known to gain insights in the process [Leitch, 1992], or to have a clue where to start. Still the key to a better understanding of the process resides within these data. In a next stage, the 
expert systems were hailed as the miracle solution for process management [Mark, 1991]. It turned out differently due to various causes. As impressive as an expert system can be, it still lacks the flexibility of a human. The knowledge in such a system is static [Rich, 1987] and doesn't adapt to different process conditions or evolutions in a plant. To keep it up to date and useful for an operator will require a lot of maintenance efforts and the expensive intervention of a knowledge engineer [Mengshoel, 1993]. Firstly one has to gather knowledge to put it into the system. This acquisition of knowledge is the acknowledged bottleneck in developing an intelligent system [Steier, 1993]. It takes up a lot of resources and the result is often poor because the knowledge engineer usually doesn't know the process [Muraret, 1993]. The presented system offers an elegant solution to these problems.

\section{GENERAL PICTURE}

The system consists of different modules. Each of them has a distinct task and can be adapted separately to specific processes and requirements.

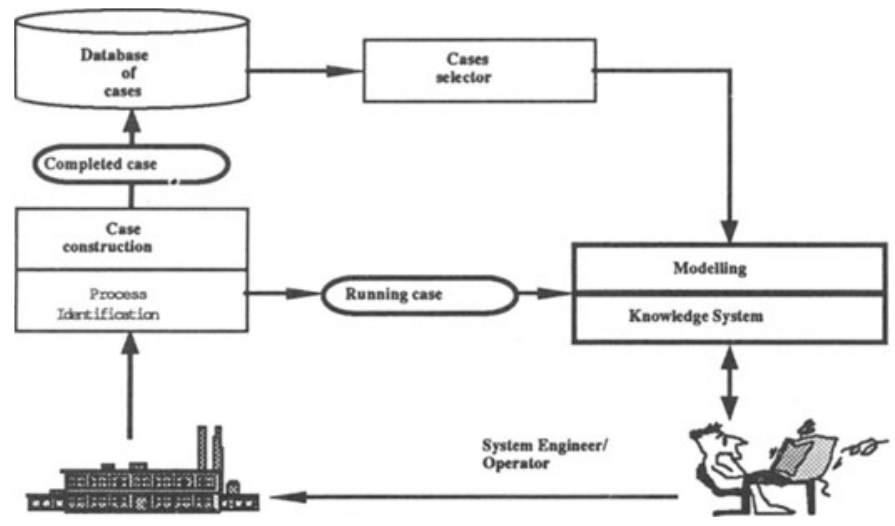

Figure 1. General architecture.

The data come from the automation system into the process identification module. This module filters out the relevant information and performs mathematical and statistical manipulations [Cheung, 1990] to come to a predefined structure: a set of process parameters that are representative for the state of the production process. We call this the running case: this is a snapshot of the current situation in the plant. Once a logical stage in the production process is reached - this of course is very process dependent -, the running case is considered complete and sent through to the database of cases. This database contains the information about all the previous plant operations structured into a standardised case form. One can search and retrieve cases out of the database through the cases selector module.

The knowledge system works with two parts: a static and a dynamic one. The static part is knowledge about your process that is already present and is considered true: it is not supposed to be changing over time. The dynamic part is knowledge that is generated on the 
fly. This dynamic knowledge is distilled out of a model. This model is build with the running case (the actual process situation) as a basis and using cases out of the database (information about previous operations). The dynamic knowledge is then confronted with the static part of the knowledge system. As a consequence the engineer working with the system can discover new knowledge and update and refine his perception of the process.

\section{CONCEPT OF A CASE}

Process information is represented in the form of cases. The purpose is to create a reference framework to organise the data in logical units. This facilitates comparing the information coming from the process with information about the previous operations in the plant. Typically a case will consist of a series of process parameters and quality parameters. Process parameters are data relating to the process under consideration. Quality parameters are constraints you want to see fulfilled at the end of your case. How you define process and quality parameters and hence your cases at hand greatly depends upon the process you are working with. Let us illustrate this with the two examples where a prototype has been installed.

The first example is a chemical pulping plant. The system is installed in the digester house. There are four digesters, each pulping wood chips with acid in a batch way. Logically you take a batch to be a case. Process parameters are everything you are logging during batch operation, including the input conditions. Quality parameters will be anything you want to achieve with the batch. The main quality parameter is here the $P$ Number (which is an indication of the amount of lignin left in the wood chips).

The second example is a plant that manufactures paper board out of waste paper. The process is a continuous one. However the paper is winded on rolls and every 20 minutes a roll is finished and a new one is started without interruption of the machinery. The production of a roll was taken as the definition of a case: this was even more obvious since every roll was controlled on several qualities like weight and strength.

The purpose of creating cases is to be able to compare them to each other: so compare one batch to another, compare the making of one roll against another.

\section{DATA HANDLING MODULES}

The data handling modules are those modules that were designed to structure and handle the data from the automation system.

The process identification and case construction module have the task to shape the data collected about the process into the case form as seen previously. The process parameters you want to see in the case might not be readily available from the automation system. To represent your process in a case you might need averages of measured values, evolutions of set points, historical data, combinations of parameters, ...[Cheung, 1990]. Also the issues of missing values and faulty measurements can be addressed at this level [Qin, 1993]. The process identification module takes care of the necessary manipulations, calculations and statistical processing of the data from the automation system to build a case. As soon as all process data are filled in and the quality parameters are available (this may take a while when 
laboratory analyses are involved) the case is considered complete and shipped to the database of cases. The process identification produces also the running case during the processing of the case. The easiest way to imagine this is as an empty skeleton of a complete case where gradually as the process continues values of process parameters are filled in. This running case must give the knowledge system an idea of the situation in the plant and will form the basis for the modelling.

The database of cases contains all the cases collected during operations in the plant. You might consider this as the memory the system has about what occurred in the plant. It is the information that implicitly lies in these past experiences that will be used by the modelling and the knowledge system to generate and validate knowledge concerning the present situation [Barletta, 1993].

The case selector module acts as a support to the modelling module in that it provides a set of cases from the database requested by the modelling. Basically the modelling asks for cases that are in some way close to the running case in order to construct a reliable model. Hence this module is able to scale and quickly retrieve cases from the database that comply to a certain criterion of nearestness to the running case. We defined the degree of nearestness as the sum of least squares of differences between a set of selected parameters, complemented with weight factors to put emphasis on more important parameters and with a correction to get a spatially uniform distribution [Loossens, 1995].

\section{MODELLING}

The modelling part of the system provides the knowledge system with a process model that can be used to derive process behaviour (the so-called knowledge rules) in a quantitative manner [Stephanopoulos, 1990]. Two modelling approaches were chosen: a linear approach (using the Partial Least Squares technique), and a non-linear approach using a backpropagation neural network [Rumelhart, 1986]. However, any modelling technique can be used (physical models about the process as well as mathematical models), since the modelling can be considered as a black-box as far as the knowledge system is concerned. Purpose of the modelling essentially is to produce relations between process and quality variables.

With the Partial Least Squares modelling approach [Geladi, 1986], we use a set of so-called nearest cases to develop a linearised system behaviour around the current point of operation of the process, hoping that the effects of the process parameters towards the qualities will be rather linear. The first tests at the industrial sites however showed that real processes rarely behave in a linear manner, so results may be completely out of scope. And even so, the behaviour is only known in the exact point of operation, where the linearisation took place so no boundary influence limits (parameter limits within which the obtained model is valid) can be calculated.

Using the Neural Network as the modelling tool requires two steps. The first step is building the model: the so-called training of the neural network with historical data. The second step is using the network to derive the process's behaviour around the current running case. In order to train the network to identify general behaviour, one can use all data logged in the database in a recent history. When one wants to look at specific behaviour (different input, start-up conditions, special production series,...), one can select only those cases that 
comply to these conditions to train the network to model this specific behaviour. The neural network we developed makes training easy for the novice user, as well as allows the experienced user to play extensively with the internal variables of the network. The result of the training contains the internal configuration of the neural network, as well as the prediction error for each case in the used dataset. The user can easily use these results to determine if neural network training was successful of not.

Initially we use the neural network to give an idea of the process evolution when changing a process parameter [Catfolis, 1994]. And this is exactly the kind of information both the system engineer and the operator is interested in. The network determines the direction of change, the range of within which the direction of change remains the same, and the order of magnitude of this change from the current point of operation onwards. These relations will be used in a qualitative manner by the knowledge system. To get an idea of the reliability of the network we used it also to predict the outcome of quality parameters. Our experiences with the prototypes installed at the industrial partners show that the neural network follows the system behaviour over a broad range (Figure 2). This strengthens our confidence in this modelling technique and motivates further investigation into this quantitative way of data mining [Loossens, 1995].

real verase predlcled CMT

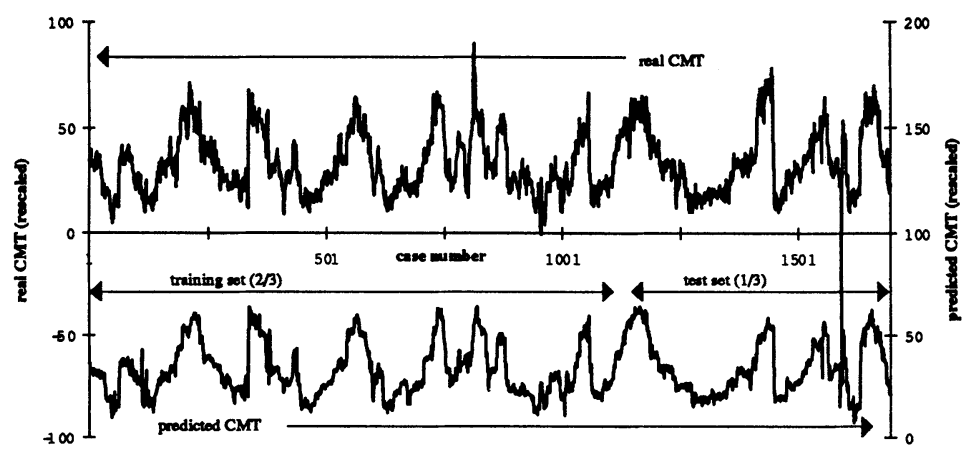

Figure 2: NN result for quality parameter CMT on the paper machine.

\section{KNOWLEDGE PROCESSING}

\section{Dynamic rule construction}

First the knowledge system converts the relation between the selected process parameters and quality parameters from the modelling into a knowledge form that is comprehensible for humans yet easy to handle by a computer. For this purpose we developed a general rule-like structure. For example, the neural net approach results in rules of the following structure:
IF <parameter_Pi $>$ changes with
<increment_Pi>, THEN
$<$ quality_Qi> will change with
<increment_Qi>

Validity of direction of change from <lower_limit_Pi $>$ to <upper_limit_Pi $>$. 
These are essentially one-to-one relations, but if a user decides to produce multiple relations (by adapting the neural network modalities) or incorporate a totally different type of modelling the rule construction is able to cope with this. Each time the modelling is triggered, the rule construction produces a set of rules. As a result we have generated knowledge about our process, not having to go through the tedious process of knowledge acquisition, but simply by taking the current situation in the plant and using previous data to derive knowledge on the fly.

\section{Dynamic rule classification}

After generating the dynamic knowledge we have to filter it through the static knowledge base (static because it is supposed to be well known and true in all circumstances). The rules that are constructed from the modelling are catalogued in three ways:

- Rules found in the static knowledge base: these are rules from the modelling that qualitatively match those from the static knowledge base. Qualitatively means that the direction of change is the same irrespectively of the absolute value of the change. We also keep track of how many times a rule in the static knowledge base has been validated and rejected. The user can consult this to quickly have a grasp as what the evolution is of his static knowledge base.

- Rules in contradiction with a rule from the static knowledge base. These are rules found by the modelling that match a rule from the static knowledge base but where the direction of change is opposite to the one in the static knowledge base. This means that the model we built of the process tells us something that is the contrary of what we think is true for the process. These rules are stored and referenced.

- Rules that are not found in the static knowledge base. This comprises in the beginning of the majority of the rules: these are the rules found by the modelling that do not match any of the rules in the knowledge base. These are logged in a separate rule base.

\section{Validation of static knowledge}

The engineer can browse through the three rule bases. In the static knowledge base he can check how many times the rules have been validated. An often validated rule means that what the modelling found is what our initial idea of the process was. The engineer can then augment the priority of this rule. This priority will be of influence on the way the operator at the control panels is presented with process knowledge from the static knowledge base.

The rule base of contradictory rules contains rules which have been found to be opposite of what the engineer's idea of the process was. He can use the knowledge system to easily backtrack to the conditions when this occurred. Different scenario's are plausible.

- An abnormal process condition occurred; a condition of which we did not have any knowledge in the static database. It is quite possible to find then that rules for normal process conditions are no longer valid. So this learns the engineer how the process behaves in these circumstances.

- The rule in the knowledge base is too general: it seems that the rule as it is stated is only valid given that certain parameters remain within certain limits. For example the rule:

IF <parameter_P1> changes with <increment_P1 > THEN

$<$ quality_Q1> will change with <increment_Q1> 
appears to be correct when parameter P1 lies between 0 and 50 whereas when $\mathrm{P} 1$ is between 50 and 100, the opposite behaviour is observed. This indicates that this rule in the static knowledge base should be adapted and expanded into two rules.

- The process is evolving, due to machine wear, replacement of machinery, different inputs, change in process management, ... As a result the knowledge about the system will be evolving and gradually you will see that some rules that were valid before are becoming more and more invalid.

- The rules in the static knowledge base were wrong in the first place. Perhaps the idea about the process was wrong.

With the knowledge system, the engineer has an intuitive tool to refine his perception of the process under his supervision and keep track of changes in the plant. The system has a user friendly interface so no extensive training is required.

\section{Other functionalities}

The knowledge system constantly generates new knowledge in the rule base of new rules. The engineer who is familiar with the process can easily distinguish between rules that don't make sense and the ones that are sensible. To test his newly gained knowledge he can put them in the static knowledge base (with a low priority) to see if they are validated in time. With the database of cases (past experiences) at his disposal, he can focus his research on a particular point of interest of his process (some correlation he suspects to exist, distinct process parts or conditions) by adapting the modelling. Hence the system skips any forgoing acquisition of knowledge.

Also the operator can benefit from this system. What the operator has at his disposal is the proofed knowledge (with a priority that is high enough) which he can consult in two ways. First he might be confronted with a deterioration of a certain quality parameter. This could be that the parameter is slowly shifting or that he gets a warning from the laboratory that the quality has turned bad. He can then query the knowledge system to see what he can do to adjust the quality. Secondly he might wonder what the effect would be if he was to change some parameter setting. The system will reason through the knowledge base to give him the implications of that change in parameter settings on the quality parameters.

Future development includes putting the system on-line: we then use the neural network model to predict the outcome (quality) of the process and in case of a bad prospect, the knowledge system uses the information from the network to advise the operator to the best corrective action(s).

\section{CONCLUSION}

We have presented a modular architecture that is able to extract and validate knowledge out of data collected from the process in the past and based on the current situation in the plant. This system provides the process engineer with a comprehensive tool to maintain and keep up to date with the knowledge about his process. Besides this it offers support to the operator to better manage his task. At the paper manufacturer, all known correlations have been confirmed and already one unknown relation has been revealed. At the pulp mill, there were at the time of writing not enough cases collected to produce reliable results (a batch takes $+/$ $10 \mathrm{hrs}$.), but the first tests already confirm the known relations. 


\section{Acknowledgement}

This architecture has been developed within the CLEAN P5285 project. This is an industrial project with 5 partners sponsored by the EC that resorts under the BRITE-EURAM program.

The authors are researchers at the Katholieke Universiteit Leuven (Belgium), in the Expert Systems Applications Development Group (part of the department of Chemical Engineering), headed by Prof. dr. ir. M. Rijckaert.

\section{References}

Barletta R. (1993) Case-based reasoning and information retrieval: opportunities for technology sharing. IEEE Expert, October, 2-4.

Catfolis, T. (1994) Generating Adaptive Models of Dynamic Systems with Recurrent Neural Networks. Proceedings of the IEEE International Conference on Neural Networks '94, vol. 5, 3238-3243, Orlando, Florida.

Cheung J. T.-Y.and Stephanopoulos G. (1990) Representation of process trends - part II: the problem of scale and qualitative scaling. Computers and Chemical Engineering, Vol. 14, No. 4/5, 511-539.

Geladi P. and Kowalski B.R. (1986) Partial least squares regression: a tutorial. Analytica Chimica Acta, 185, 1-17.

Leitch R. (1992) Artificial intelligence in control: some myths, some fears but plenty of prospects. Computers \& Control Engineering Journal, July, 153-163.

Loossens K. and Van Houtte G. (1995) Predicting Process Evolution with a Neural Network. A window on relevant data. Proceedings of the IEEE Industrial Automation and Control Conference '95, Taipei, Taiwan.

Mark W.S. and Simpson R.L.Jr. (1991) Knowledge-based systems: an overview. IEEE Expert, June, 12-17.

Mengshoel O. J. and Delab S. (1993) Knowledge validation: principles and practice. IEEE Expert, June, 62-68.

Muratet G.and Bourseau P. (1993) Artificial intelligence for process engineering - state of the art. Computers and Chemical Engineernig - special issue: European symposium on computer aided process engineering, February, S381-S385.

Qin, S. J. and Rajagopal, B. (1993) Combining statistics and expert systems with neural networks for empirical process modelling. Advances in Instrumentation and Control, Vol. 48, part 3, 1711-1720.

Rich S.H. and Venkatasubramanian V. (1987) Model-based reasoning in diagnostic expert systems for chemical process plants; Computers and Chemical Engineering, Vol. 11, No. 2, 111-122.

Rumelhart, D.E., Hinton, G.E. and Williams, R.J. (1986) Learning internal representations by error propagation. In D.E. Rumelhart, JL. McClelland and the PDP Research group (eds.) Parallel Distributed Processing: Explorations in the Microstructure of Cognition, Volume 1: Foundations.

Steier, D. M. et al. (1993) Combining multiple knowledge sources in an integrated intelligent system. IEEE Expert, June, 35-44.

Stephanopoulos G. (1990) Artificial intelligence in process engineering - current state and future trends. Computers and Chemical Engineering., Vol. 14, No. 11, 1259-1270. 